\title{
Casos Clínicos
}

\section{Manejo de una paciente embarazada con déficit congénito de Factor VII leve}

\author{
Nigel P Murray ${ }^{1,2}$, Javier llabaca ${ }^{3}$ Néstor Lagos ${ }^{3}$, Miguel Biron $^{3}$, Cristian Pinats ${ }^{3}$, Simona \\ Minzer ${ }^{4}$.
}

\author{
${ }^{1}$ Hematologo, Servicio de Medicina, Hospital de Carabineros de Chile, Simón Bolívar 2200, Nuñoa, Santiago. \\ 2 Profesor de Hematología, Facultad de Medicina, Universidad Finís Terrae, Av. Pedro de Valdivia 1509, \\ Providencia, Santiago. \\ ${ }^{3}$ Servicio de Obstétrica y Ginecología, Hospital de Carabineros de Chile, Simón Bolívar 2200, Nuñoa, Santiago. \\ ${ }^{4}$ Servico de Medicina, Hospital de Carabineros de Chile, Simón Bolívar 2200, Nuñoa, Santiago.
}

\section{RESUMEN}

No hay guías específicas para el manejo de pacientes embarazadas con la deficiencia de Factor VII ; no hay una correlación entre el nivel de FVII y el riesgo de hemorragia y el nivel del Factor VII aumento durante el embarazo. Presentamos un caso clínico, el manejo y las recomendaciones del consenso.

Palabras clave: Deficiencia de Factor VII, embarazo; manejo.

\section{INTRODUCCIÓN}

Las hemorragias durante el embarazo, postparto inmediato y puerperio tardío son complicaciones que trae una morbididad y mortalidad a la paciente y el feto. Informes internacionales reportan una incidencia de $1.2 \%$ de hemorragias durante el embarazo, $8.3 \%$ en el post-parto inmediato con parto vaginal y un $5.7 \%$ pos-cesárea y $0.2 \%$ en el puerperio tardío $1-3$. Sin embargo, la incidencia de transfusión fue de 1,14\% 2 . Los procedimientos quirúrgicos obstétricos se asocian con sangrado post operatorio, el cual es habitualmente auto-limitado. No obstante, en pacientes con desórdenes hereditarios de la hemostasia, procedimientos invasivos menores pueden precipitar sangrados prolongados4. Estos sangrados excesivos son alarmantes tanto para el paciente como para el tratante, y pueden alargar el procedimiento, comprometer la cicatrización y predisponer a infecciones.

El déficit congénito de factor VII es una condición autosómica recesiva que produce un déficit severo en el homocigoto y un déficit moderado sin tendencia al sangrado en el heterocigoto con una incidencia estimada de 1 en 500.000 en caucásicos. El paciente homocigoto típicamente tiene niveles del Factor FVII menor de $10 \%$ mientras para los heterocigotos el nivel del Factor VII es entre $20-60 \% .4$ Sin embargo, los fenotipos clínicos varían desde asintomático, incluyendo en homocigotos y heterocigotos dobles a pacientes con una enfermedad hemorrágica severa5. Pacientes homocigóticas por la misma mutación pueden tener diferencias significativas en el fenotipo clínico. Por lo tanto, el estudio genético no es de utilidad en el manejo clínico del paciente. En el déficit severo de factor VII, los niveles no pueden determinar de manera confiable las manifestaciones clínicas. Los pacientes están habitualmente asintomáticos, pero pueden sangrar durante procedimientos invasivos. Sangrados severos tienden a ocurrir en individuos con un nivel de actividad del factor VII de $2 \%$ o menor al valor normal. Otras presentaciones clínicas incluyen epistaxis espontánea, sangrado subcutáneo profundo, hemorragias gastrointestinales 0 genitourinarias y hemartrosis, las cuales son manifestaciones comunes pero que comúnmente pueden ocasionar sangrados mortales4. En la clínica, un nivel del Factor VII de $15-20 \%$ es utilizado como un punto de corte para considerar terapia de reemplazo, pacientes con un nivel de Factor VII mayor de $20 \%$ no 
debieran sangrar, en el caso de una historia clínica previa de hemorragia en estés pacientes es importante descartar otro defecto de la coagulación.

Presentamos el caso de una mujer de 20 años embarazada con déficit congénito de factor VII leve, se discute el manejo del caso y se destacan puntos de aprendizaje con respecto al reemplazo de factor VII y riesgo de sangrado.

Caso Clínico: Una mujer de 20 años, primigesta, se presentó con un embarazo de 9 semanas, confirmado por ultrasonido. A los 8 años de edad fue evaluada para someterse a adenoamigdalectomía; no presentada historia personal ni familiar de sangrado o coagulopatías. Dentro de los exámenes de laboratorio pre-quirúrgicos se observó un tiempo de protrombina (TP) de 18 segundos (rango normal 11-13 segundos) o $50 \%$ (rango normal $70-100 \%$ ), y un tiempo de tromboplastina parcial activada (TTPA) normal. EI TP prolongado no fue corregido con vitamina $K$ intravenosa (30 mg en total) y las pruebas de función hepática fueron normales. Los niveles de Factor VII (FVII) fueron 48\% (rango 60-160\%) por lo que se diagnosticó déficit de FVII. Recibió 2 unidades de PFC pre $y$ en el post-quirúrgico inmediato, sin complicaciones hemorrágicas.

A los 13 años la paciente fue sometida a una extracción dental, por lo que nuevamente se trató con PFC. A esta altura, la paciente había sido diagnosticada de hipotiroidismo y resistencia a la insulina y estaba siendo tratada con tiroxina $y$ metformina. No hubo episodios hemorragíparos, sus menstruaciones eran normales y no abundantes y no había presentado abortos.

A la semana 9 de embarazo, su TP fue de $48 \%$ y los niveles de FVII fueron de $49 \%$, consistente con un déficit leve de FVII. No se presentaron inconvenientes durante el embarazo y a las 38 semanas el TP aumentó hasta $87 \%$, con niveles de FVII de $114 \%$. No se consideró profilaxis ya que el riesgo de sangrado se normalizó. A las 40 +2 semanas de embarazo, el registro basal no estresante fue normal pero no hubo contracciones uterinas, la paciente tuvo una inducción del parto con oxitócina. Por falta de dilatación cervical fue sometida a una cesárea, no hubo sufrimiento fetal. Se administró anestesia epidural. Se utilizó carbetocina y metilergonovina posterior a la cesárea para estimular la contracción uterina. La paciente no tuvo sangrado excesivo, no requirió hemocomponentes en términos de PFC o transfusiones de glóbulos rojos y por lo tanto fue dada de alta al tercer día post operatorio. El recién nacido fue masculino, de 3,200gm, medio $49 \mathrm{~cm}$ con puntaje de APGAR de 9 y 9.

Discusión: El déficit de FVII aislado está siendo más frecuentemente diagnosticado dado la mejoría en el tamizaje pre-operatorio. Sin embargo, no hay guías específicas para el manejo de estos pacientes. El déficit de FVII se clasifica en tipo I (ausencia de FVII) o tipo II (presencia de FVII disfuncional). El gen del FVII está ubicado en el cromosoma 13 y se han descrito más de 130 mutaciones y la correlación entre el nivel de FVII circulante y el nivel de sangrado no es buena, a diferencia del déficit de FVIII o FIX, en los cuales la severidad del sangrado se asocia directamente con el nivel de actividad del factor5.

Hay una tendencia que el riesgo de una hemorragia aumenta con la disminución del nivel de Factor VII sin embargo e importantemente no hay un punto de corte que separar los pacientes "hemorrágicos" de los "no-hemorrágicos". Utilizando un punto de corte del nivel de Factor VII sérico de $20 \%$ de normal, una revisión sistémica determino que la profilaxis con Factor VII solo si utilizo en un tercero de casos; la frecuencia de hemorragias fueron semejantes entre las pacientes tratadas con profilaxis $y$ en aquellos que no tuvieron profilaxis; niveles de Factor VII sérico fueron similares entre pacientes con o sin hemorragia y con aquellos con o sin una historia previa de hemorragia4. Sin embargo, hay un consenso que un paciente con niveles séricos de Factor VII menor que $20 \%$ debe tener profilaxis6. En este mismo consenso se recomienda que individuos con historia personal de sangrado quirúrgico y un nivel de FVII mayor a $20 \%$ y también aquellos con historia familiar de sangrado y un FVII menor a $20 \%$ deben recibir profilaxis6.

Es importante notar que en el Registro Internacional de Déficit Congénito de FVII, las mujeres fueron sintomáticas más frecuentemente que los hombres, con una mayor prevalencia de sangrado muco-cutáneo, con la presencia de menorragia en un $46 \%$ de las pacientes y en el $12 \%$ siendo el síntoma inicial. La presencia de sangrado muco-cutáneo fue un factor predictivo para sangrado ginecológico 7 .

Durante el embarazo hay un aumento fisiológico de los niveles de Factor VII, especialmente en el tercer trimestre, como consecuencia en pacientes con una deficiencia leve o moderada el nivel del FVII podría aumentar hasta niveles normales y protege contra hemorragia excesiva, como en el caso reportado. En el grupo de pacientes con una deficiencia leve a moderada el riesgo de sangrado es mayor en el primer trimestre mientras los niveles de Factor VII aún son 
bajos siendo incluso causa de aborto8. En pacientes con una deficiencia severa, esta respuesta fisiológica no se ocurre. Por lo tanto, es necesario medir los niveles del Factor VII en el tercer trimestre para planificar el parto, vaginal o cesárea8.

La decisión para proceder a parto vaginal o cesárea es puramente obstétrica, sin embargo, según la literatura pacientes debe ser considerados para profilaxis con Factor VII4. El número limitado de pacientes con deficiencia de Factor VII y hemorragia post-cesárea significa que la seguridad del paciente sin el uso de profilaxis no puede ser garantizado.

Si tratamiento está indicado, el uso de plasma fresca congelada en una dosis de $15-30 \mathrm{ml} / \mathrm{kg}$, dado su amplia disponibilidad, bajo potencial trombogénico y relativo bajo costo sigue ser utilizado en algunos países. El uso de concentrados de FVII con una dosis de 40IU/kg o como una dosis fija según el tamaño de la ampolla del fármaco según la disponibilidad del fármaco ha sido utilizado. Sin embargo, ninguno de estos dos productos es viralmente inactivados, el riesgo de la transmisión viral es mucho más elevado en el FVII concentrado que la plasma fresca congelado, pero con ambos productos hay un riesgo de la transmisión viral al paciente o al feto. La vida media corta (3-4 horas) del Factor VII significa que se requerir de altos volúmenes de plasma.

El uso del Factor VIla activado recombinante (rFVIla) no tiene riesgo de la transmisión viral y no hay el problema de sobrecarga de volumen. Sin embargo, es de alto costo y no todos los pacientes tienen acceso. Tampoco hay consenso en la dosis más adecuado, la dosis recomendada para tratar hemorragias en pacientes con deficiencia de Factor VII es de $15-30 \mathrm{~g} / \mathrm{kg}$ cada 4-6 horas. Para el uso profiláctico no hay recomendaciones claras, Loddo et al9 recomendó una dosis de $20 \mathrm{~g} / \mathrm{kg}$ al tiempo de la dilatación cervical completo y una segunda dosis cuatro horas más tarde en el caso del parto vaginal. Otro autores han usado diferentes dosis, desde $50 \mathrm{~g} / \mathrm{kg}$ al tiempo de la dilatación completa y una segunda dosis de $35 \mathrm{~g} / \mathrm{kg}$ cuatro horas más tarde hasta la más frecuente de $20-30 \mathrm{~g} / \mathrm{kg}$ en la primera dosis y seguido por la misma dosis cuatro horas más tarde9. En el caso de una cesárea, se recomienda la primera dosis 30-60 minutos antes de la cirugía y cada cuatro horas durante el periodo máximo de riesgo de hemorragia9. Este significa que la paciente debe ser monitorizada para identificar hemorragias y permitir la terapia de reemplazo precozmente.

El uso de agentes anti-fibrinolíticos como el ácido tranexámico para la prevención de hemorragia después del parto ha sido reportado a disminuye el riesgo de hemorragia postparto 10 . No es de uso rutina y no hay evidencia de su beneficio en pacientes con deficiencia de Factor VII, por parto vaginal o por cesárea. A revisión sistémica por Cochrane concluyó que cuando administrado en forma endovenosa el ácido tranexámico disminuyo la mortalidad por hemorragia postparto 11. Recomendamos que su uso debe ser considerado solamente en caso de hemorragia post-parto.

Con la corrección de la coagulopatía, hay unl riesgo de trombosis, que se estima entre 1 por cada 1,000-2,000 embarazos. El riesgo de trombosis es muy bajo $(<0,4 \%)$ con el uso de rFVIla en una dosis de $20-30 \mathrm{~g} / \mathrm{kg} 9$. El uso de medias antitrombóticas, movilización precoz y post cirugía profilaxis con heparina de bajo peso molecular son recomendadas.

En este reporte de caso, el uso de PFC para cirugías previas probablemente no estaba indicado; el miedo al sangrado por el personal médico puede llevar a tratamiento inapropiado. En pacientes con TP prolongado por enfermedad hepática, déficit de vitamina $\mathrm{K}$ o uso de anticoagulantes antagonistas de vitamina $\mathrm{K}$, en los cuales existe un déficit de FVII, FII, FIX y FX, el riesgo de sangrado varía según la prolongación del TP, a diferencia de los pacientes con déficit aislado de FVII. Como tal, puede ocurrir un sobre tratamiento con hemocomponentes, utilizando productos que no han sido sometidos a un proceso de inactivación.

Se recomienda que mujeres con déficit de FVII leve a moderado, sin historia previa de sangrado personal o familiar, se deben medir niveles de FVII al diagnóstico y durante el tercer trimestre del embarazo. Aquellas pacientes con niveles de FVII mayores a $20 \%$, deben ser observadas y tener acceso a terapia de reemplazo en caso de ser necesario. Las pacientes que presenten niveles de FVII menores a $20 \%$, se debe considerar profilaxis con FVIla o PFC, especialmente en casos de cesárea. El uso de ácido tranexámico es controversial, pero podría ser considerado en caso de hemorragia activa.

Se recomienda manejo interdisciplinario entre obstetricia, hematología y anestesia, individualizado para cada paciente.

Conflictos de interés: Los autores no tienen conflictos de interés.

Agradecimientos: Sra. Ana Maria Palazuelos por su ayuda en la redacción de este manuscrito.

Consideraciones éticas: El caso clínico fue aprobado por el Comité Ética del Hospital de 
Carabineros de Chile conforme con la ley chilena vigente.

\section{BIBLIOGRAFÍA}

1. Giordano R, Cacciatore A, Cignini P, Vigna R, Romano M. Antepartum haemorrhage. J Prenatal Med 2010; 4: 12-16.

2. Jegasothy E, Patterson J, Randall D, Nippita TA, Simpson JM, Irving DO et al. Assessing the effect of risk factors on rates of obstetric transfusion over time using two methological approaches. BMC Med Res Methodol 2018; doi: 10.1186/s12874018-0595-6

3. Fein A, Wen T, wright JD, Goffman D, D'Alton ME, Attenello $\mathrm{FJ}$ et al. Postpartum hemorrhage and risk for postpartum readmission. J Matern Fetal Neonatal Med 2019; 9: 1-8

4. Baumann-Kreuziger LM, Morton CT, Reding MT. Is prophylaxis required for delivery in women with factor VII deficiency? Haemophilia 2013; doi:10.11/hae.12167

5. Mariani G, Hermann FH, Dolce A, Batorova A, Etro $D$, Pewyvandi $F$ et al. Clinical phenotypes and factor VII genotype in congential factor VII deficiency. Thromb Haemost 2005; 93: 481-487

6. Sheth S, Soff G, Mitchell B, Green D, Kaicker S, Fireman $\mathrm{F}$ et al. Managing incidentally diagnosed isolated Factor VII deficiency perioperatively: a brief expert consensus report. Expert Rev Hematol 2012; 5: 47-50

7. Lapecorella M, Mariani G. International Registry on Congenital Factor VII Deficiency. Factor VII deficiency: defining the clinical picture and optimizing therapeutic options. Haemophilia 2008; 14: $1170-1175$

8. Kulkarni AA, Lee CA, Kadir RA. Pregnancy in women with congenital factor VII deficiency. Haemophilia 2006; 12: 413-416

9. Loddo A, Cornacchia S, Lo Cane F, Barcellona D, Marongiu F, Melis GB et al. Prophylaxis of peripartum haemorrhage using recombinant factor VIla (rfVIla) in pregnant women with congenital factor VII deficiency: a case report and literatue review. Eur J Obstet Gynecol \& Reprod Biol 2019; 235: $77-80$

10. Sentilhes L, Winer N, Azria E, Senat MV, Le Ray $C$, Vardon D et al. Tranexamic acid for the prevention of blood loss after vaginal deli very. $\mathrm{N}$ Eng J Med 2018; 379: 731-742

11. Shakur H, Beaumont D, Pavord S, Gayet-Ageron A, Ker K, Mousa HA. Antifibrinolytic drugs for treating primary postpartum heaemorrhage. Cochrane Database Syst Rev. 2018; doi:10.1002/14651858.CD012964 